\title{
Patterns of International Author Collaboration in Surgery
}

\author{
Chien TW' ${ }^{1}$ Chang $\mathrm{Y}^{2}$, Chow $\mathrm{J} \mathrm{C}^{3}$ and Chou $\mathrm{W}^{4,5 *}$ \\ ${ }^{1}$ Research Department, Chi-Mei Medical Center, Taiwan \\ ${ }^{2}$ National Taiwan University School of Medicine, Taiwan \\ ${ }^{3}$ Department of Pediatrics, Chi Mei Medical center, \\ Taiwan \\ ${ }^{4}$ Department of Physical Medicine and Rehabilitation, Chi \\ Mei Medical Center, Tainan \\ ${ }^{5}$ Department of Recreation and Health-Care Management \\ $\&$ Institute of recreation, Industry Management, Chia \\ Nan University of Pharmacy, Taiwan \\ *Correspondling author: Willy Chou, Department of \\ Recreation and Health-Care Management \& Institute of \\ Recreation Industry Management, Chia Nan University of \\ Pharmacy, Taiwan
}

Received: November 23, 2017; Accepted: December 15, 2017; Published: December 22, 2017

\section{Abstract}

Objective: To investigate patterns of international author collaborations in surgery by collecting data from Medline and to visualize data using Google maps and Social Network Analysis (SNA)

Methods: Selecting 993 abstracts, author names, countries, and Medical Subject Headings (MESH) on December 2, 2017 from Medline based on journal of surgery in recent three years, we reported following features: (1) nation distribution for 1st author's nationality; (2) prominent authors in the field of surgery, and (3) research features represented by paper's MESH terms. We programmed Microsoft Excel VBA routines to extract data from Medline. Google Maps and SNAP ajek software were performed to display graphical representations with an easy-to-read feature for readers.

Results: We found that (1) the most number of papers in surgery are from nations of U.S. $(413,41.59 \%)$ and Japan $(115,11.58 \%)$; (2) the proactive authors in surgery are Hiroaki Nagano and Keith DLillemoe; (3) the most linked MESH terms are hepatectomy/adverse effects/methods and portal vein/anatomy \& histology/diagnostic imaging/surgery.

Conclusion: Social network analysis provides wide and deep insight into the relationships among nations, coauthor collaborations, and MESH terms. The results can be provided to readers for future submission to journal in surgery.

Keywords: Abstract keywords; Authorship collaboration; Google Maps; Social network analysis; Medline

\section{Introduction}

Surgery is a medical specialty that uses operative manual and instrumental techniques on a patient to investigate (or treat a pathological condition such as a disease or injury) to help improve bodily function or appearance or repair unwanted ruptured areas [1]. Many journals are included in surgery such as cardiac surgery, cardiothoracic surgery, colorectal surgery, endocrine surgery, general surgery, neurosurgery, obstetrics and gynecologic surgery, surgical oncology, ophthalmic surgery, oral and maxillofacial surgery, orthopedic surgery, head and neck surgery, pediatric surgery, plastic surgery, transplant surgery, thoracic surgery, vascular surgery, digestive surgery, trauma surgery, acute care surgery, laparoscopic surgery, bariatric and GI surgery, colorectal surgery, foregut surgery, colon and rectal Surgery, et al. Which nation plays the most important role in surgery is required to report. Meanwhile, which author published most papers in academics and which research domain is most prevalent in recent years are worthwhile to investigate.

In real world, any entity is rare independent in existence. Comorbid is defined in medicine as existing simultaneously with and usually being co-occurred with one another, such as screw sagittal angle related to stress on endplate of adjacent segments and lymph node metastasis associated with a gastric cancer patient $[2,3]$. In many situations, it is very hard to observe the association of two or more symptoms or entities existed in a system at a moment.

An apocryphal story often told to illustrate the concept of cooccurrence is about beer and diaper sales. It usually goes along with both beer and diaper sales which were strongly correlated [4-6] in a supermarket. All possible pairs of our observed objects can be combined to examine by using computer algorithms. However, we have not seen any demonstrate how to pick up the most possible pairs co-occurred in our datasets.

\section{Social Network Analysis (SNA)}

Social Network Analysis (SNA) [7-9] has been applied to authorship collaboration in recent years. Co-authorship among researchers can form a type of social network, called co-author network [7]. We are thus interested in using SNA to explore the closest relation in surgery from data we observed in Medline library.

\section{Author collaborations and international relations}

Many papers have been collected and saved in Medline library (Pubmed.com). However, we have not seen any using Google maps to demonstrate their study results in literature even if computer scientists have put their hopes on those machine-learning algorithms, data mining or artificial intelligence to quantify research information $[10,11]$. Extracting papers from Medline is a way to release some important information in surgery using Google maps to increase the yield of knowledge from data generated in the course of inquiry [1214]. However, the messages on coauthor collaboration and the most productive author in surgery is still unclear.

\section{Aims of the study}

Our aims are to investigate patterns of international author collaborations in surgery by collecting data from Medline and to visualize results on following topics: (i) nation distribution in papers 
regarding surgery; (ii) the most prominent authors in surgery; (iii) the recent research domains defined by Medical Subject Headings (MESH) terms.

\section{Methods}

\section{Data sources}

We programmed Microsoft Excel VBA (Visual Basic for Applications) modules to extract abstracts and their corresponding coauthor names as well as MESH terms for each article on December 2, 2017 from Medicine National Institutes of Health (Medline) based on publications in recent three years. Only those abstracts published by the keyword surgery [journal] and labeled with Journal Article were included. Others like those labeled with Published Erratum, Editorial or without author nation name were excluded from this study. A total of 993 eligible abstracts were obtained from Medline since 2015.

\section{Data arrangement to fit SNA requirement}

Prior to visualize representations of interest in this study using SNA, we organized data in compliance with the SNA format and guidelines using Pajek software [15]. Microsoft Excel VBA was used to deal with data fitting to the SNA requirement.

\section{Graphical representations to report}

Author nations and their relations: A table (i.e. columns for publication years and rows for the 1st author nations) was made for presenting the distribution of nations regarding surgery. The bigger bubble means the more number of the nodes (i.e, nations, or MESH terms in this study). The wider line indicates the stronger relations between two nodes. Community clusters are filled with different colors in bubbles.

Keywords and MESH terms to present the research domain: If keywords represent the research domain, the stronger relations between two keywords can be highlighted by SNA, like the concept of co-occurrence about beer and diaper sales. The presentation for the bubble and line is interpreted similar to the previous section.

\section{Statistical tools and data analyses}

Google Maps [16] and SNAP ajek software [15] were used to display visualized representations for key authors and MESH terms in relation with surgery. Author-made Excel VBA modules were applied to organize data. Cluster coefficient represents the density of a network and is defined as CC= $\frac{\text { number of closed troplets }}{\text { number of possible connected triplets of nodes }}=\frac{\sum_{i=1}^{n} \text { other_two_nodes } \_ \text {_connected }}{\sum_{i=1}^{n}\left(m_{i}{ }^{*}\left(m_{i}-1\right) / 2\right.}$,

whereas $\mathrm{n}=$ the number of nodes in a network and $\mathrm{m}=$ the number of other connected nodes with a specific ego node. A significant lever $(>1.96)$ is defined by $t$-value as the formula $\left[=c c^{*} \sqrt{ }[(n-2) /(1-c c 2)]\right.$.

\section{Results}

\section{Author nations and their relations}

A total of 993 eligible papers with complete author nations based on journal article since 2015 are shown in (Table 1). We can see that the most number of papers are from nations of U.S. (413, 41.59\%) and Japan $(115,11.58 \%)$. The trend in the number of publications
Table 1: Nation distribution based on the $1^{\text {st }}$ author for papers published in Surgery.

\begin{tabular}{|c|c|c|c|c|c|c|}
\hline Nation & 2015 & 2016 & 2017 & Total & $\%$ & Growth \\
\hline U.S & 110 & 130 & 173 & 413 & 41.59 & 0.98 \\
\hline Japan & 31 & 44 & 40 & 115 & 11.58 & 0.68 \\
\hline China & 20 & 20 & 13 & 53 & 5.34 & -0.87 \\
\hline France & 13 & 17 & 23 & 53 & 5.34 & 0.99 \\
\hline Italy & 12 & 16 & 16 & 44 & 4.43 & 0.87 \\
\hline Netherlands & 15 & 8 & 16 & 39 & 3.93 & 0.11 \\
\hline Canada & 8 & 11 & 9 & 28 & 2.82 & 0.33 \\
\hline Germany & 7 & 10 & 11 & 28 & 2.82 & 0.96 \\
\hline U.K & 12 & 9 & 5 & 26 & 2.62 & -1 \\
\hline Spain & 3 & 3 & 12 & 18 & 1.81 & 0.87 \\
\hline Sweden & 2 & 5 & 6 & 13 & 1.31 & 0.96 \\
\hline Australia & 8 & 1 & 3 & 12 & 1.21 & -0.69 \\
\hline India & 1 & 6 & 5 & 12 & 1.21 & 0.76 \\
\hline Switzerland & 3 & 1 & 6 & 10 & 1.01 & 0.6 \\
\hline Turkey & 2 & 4 & 4 & 10 & 1.01 & 0.87 \\
\hline Greece & 2 & 3 & 4 & 9 & 0.91 & 1 \\
\hline Taiwan & 4 & 3 & 2 & 9 & 0.91 & -1 \\
\hline Austria & 1 & 5 & 2 & 8 & 0.81 & 0.24 \\
\hline Finland & 1 & 4 & 3 & 8 & 0.81 & 0.65 \\
\hline Denmark & 2 & 2 & 3 & 7 & 0.7 & 0.87 \\
\hline South Korea & 3 & & 4 & 7 & 0.7 & 0.24 \\
\hline Brazil & 1 & 2 & 3 & 6 & 0.6 & 1 \\
\hline Israel & 2 & & 4 & 6 & 0.6 & 0.5 \\
\hline Singapore & 4 & 1 & 1 & 6 & 0.6 & -0.87 \\
\hline Norway & 2 & 2 & 1 & 5 & 0.5 & -0.87 \\
\hline Egypt & 1 & 1 & 2 & 4 & 0.4 & 0.87 \\
\hline Hong Kong & & 2 & 2 & 4 & 0.4 & 0.87 \\
\hline Ireland & 1 & 1 & 2 & 4 & 0.4 & 0.87 \\
\hline Mexico & & 2 & 2 & 4 & 0.4 & 0.87 \\
\hline Hungary & & 1 & 2 & 3 & 0.3 & 1 \\
\hline Others & 6 & 12 & 11 & 29 & 2.92 & 0.78 \\
\hline Total & 277 & 326 & 390 & 993 & 100 & 1 \\
\hline
\end{tabular}

with authorship from countries is present in the column of growth in (Table 1). The diagram shown by SNA and Google Maps in (Figure 1) displays author collaboration among nations based on journal name involving surgery. The highest productive nations are from U.S. and Asia (Japan and China). Any nation collaborated with other nations are shown with a blue line. Interested authors are recommending clicking the bubble of interest to see details on a website at reference [17]. Several clusters are shown in (Figure 2), indicating that nations have a closer relation representing an identical color. The cluster represented by Sweden earns the highest density with a $\mathrm{cc}=0.84$. In contrast, the lowest $\mathrm{cc}$ is shown in the network of US and Japan with a $c c=0.0$, respectively. We can see that any two nations have not connected with each other in the two clusters represented by US and Japan. The link on website was provided at reference [18]. 


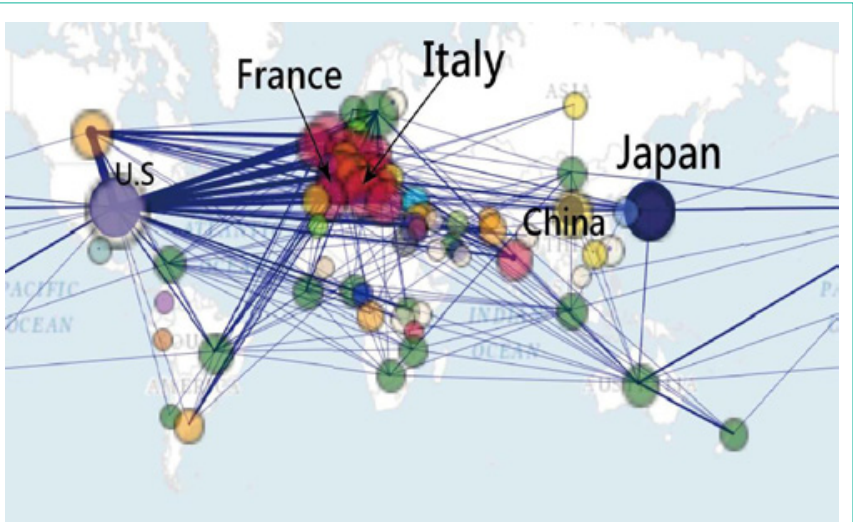

Figure 1: Top 5 nations of international coauthor collaboration in topics of Surgery.

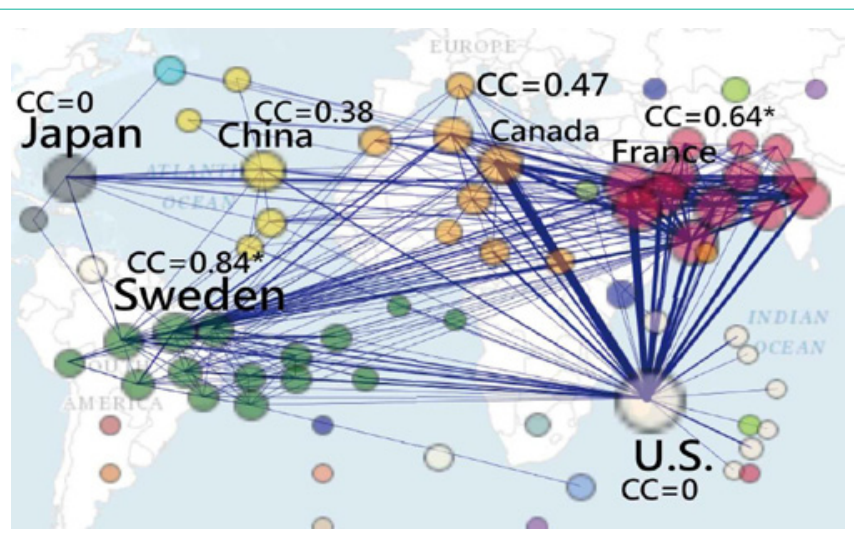

Figure 2: Cluster coefficients of international coauthor collaboration in topics of Surgery.

\section{The prominent authors in surgery}

The most prominent authors in who published most number of papers regarding surgery are Hiroaki Nagano and Keith DLillemoe, see (Figure 3). The link on website was referred to reference [19].

\section{MESH terms to present the feature of research domain recently}

The most linked MESH terms are hepatectomy/adverse effects/ methods and portal vein/anatomy \& histology/diagnostic imaging/ surgery., see (Figure 4) or click it on the reference [20]. We can see that the two bigger bubbles are the two mentioned above in the blue cluster.

\section{Discussion}

This study used SNA techniques to report that (1) the most number of papers in surgery are from nations of U.S.(413,41.59\%) and $\operatorname{Japan}(115,11.58 \%)$; (2) the proactive authors in surgery are HiroakiNagano and Keith DLillemoe; (3) the most linked MESH terms are hepatectomy/adverse effects/methods and portal vein/ anatomy \& histology/diagnostic imaging/surgery.

\section{What this adds to what was known}

Many previous researches [7-9] have investigated coauthor collaboration using SNA. The results (the most number of articles in surgery from US and Asia) are different from the findings that

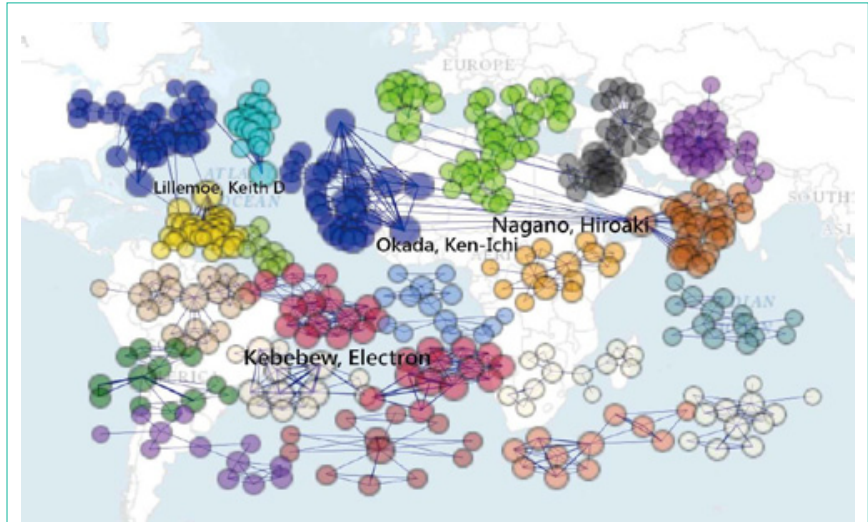

Figure 3: Prominent authors in papers in topics of Surgery.

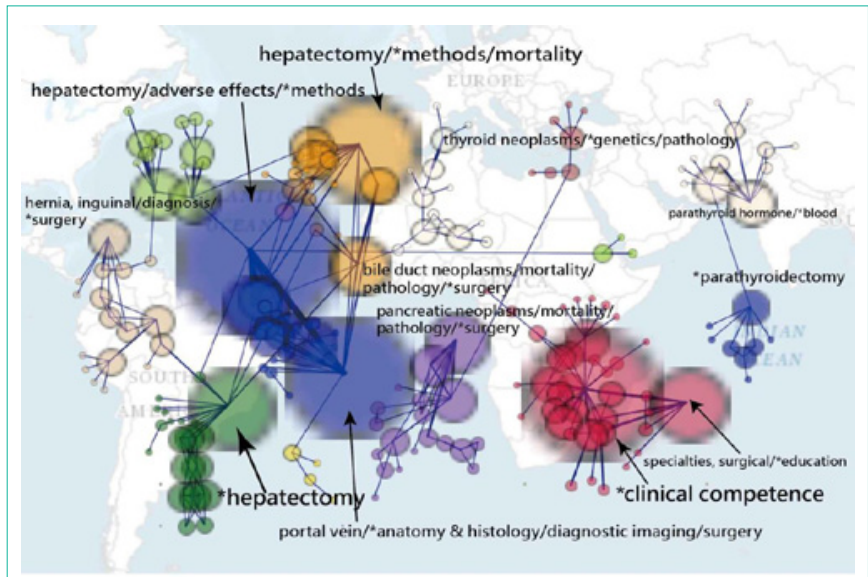

Figure 4: Mesh terms in papers regarding nursing and palliative.

dominant nations in science come from U.S. and Europe [21,22]. Back to the apocryphal story told to discover the co-occurrence about beer and diaper sales [4-6], we demonstrated a way incorporating SNA with Google maps to explore data and to present visual representations to readership which is never seen before in literature. Traditionally, it is very hard to observe the association of two or more symptoms or entities existed in a system at a moment.

Journal authorship collaboration can be compared with each other using Google Maps. We can see that many links connecting two nations which indicate a collaboration pattern in paper publication similar to the previous study [7]. Hence the researchers have a high level of international author collaboration in surgery which is inconsistent with the previous studies that investigated scientific collaboration of Iranian Psychology and Psychiatry Researchers $[23,24]$.

There are 1617 papers with the keyword social network analysis in paper title when searching Medline in 2017 December $1^{\text {st }}$. Only two papers $[25,26]$ incorporated $\mathrm{MeSH}$ into social network analysis to explore interesting knowledge. However, no any that can incorporate Google maps into a study to show information to readers. The way we illustrated the strongest relation in all possible couples of interest is novel and promising in future, especially on the topic of surgery in publish papers. 


\section{What it implies and what should be changed?}

Scientific publication is one of the objective measurements to evaluate the achievements of a medical specialty or discipline [27]. It is worth using SNA and Google Maps to explore journal features in future.

Several algorithms and measures have been developed and used with SNA to graphically explore data. If we investigate whether any author or paper most fits the research domain of a journal with the target journal's scope, the centrality measures using SNA can be applied [7]. It means that the core subject can be analyzed using the centrality measure $[24,28]$ yielded in SNA.

\section{Strengths of this study}

The way incorporating SNA with Google Maps is unique, which is never seen in other published papers [7-9] with a single SNA presentation. Another strength and feature for this study is the Google Maps used and linked in references [17-20] for interested readers who can quickly understand the features of the study. The nation distribution in (Figure 1) is merit in easily understanding the feature of international author collaborations on the topic of surgery. One picture is worth ten thousand words. We hope following studies can report more such kinds of information using SNA and Google Maps to readers in future.

\section{Limitations and Future Study}

The interpretation and generalization of the conclusions of this study should be carried out with caution. First, the data of this study were collected from Medline for a single journal. It is worth noting that any attempt to generalize the findings of this study should be made in the similar journal domain with similar topic and scope contexts.

Second, although the data were extracted from Medline and carefully dealt with every linkage as correct as possible, the original downloaded text file including some errors in symbols such as period and comma in author address that might lead to some bias in the resulting nation distribution.

Third, there are many algorithms used for SNA. We merely applied separation components showing in Figures. Any changes made along with algorithm used will present different pattern and judgment.

Fourth, the social network analysis is not subject to the Pajeck software we used in this study, others such as Ucinet [29] and Gephi [30] are suggested to readers for use in future.

\section{Conclusion}

Social network analysis provides wide and deep insight into the relationships among nations, coauthor collaborations, and MESH terms. The results can be provided to readers for future submission to journal in surgery.

\section{References}

1. Koch M, Garden OJ, Padbury R, Rahbari NN, Adam R, Capussotti L, et al. Bile leakage after hepatobiliary and pancreatic surgery: a definition and grading of severity by the International Study Group of Liver Surgery. Surgery. 2011; 149: $680-688$.

2. Zhang $\mathrm{Y}$, Tang $\mathrm{Y}$, Shen $\mathrm{H}$. Relationship between screw sagittal angle and stress on endplate of adjacent segments after anterior cervical corpectomy and fusion with internal fixation: a Chinese finite element study. BMC Surg. 2017; 17: 119

3. Bausys R, Bausys A, Vysniauskaite I, Maneikis K, Klimas D, Luksta M, et al. Risk factors for lymph node metastasis in early gastric cancer patients: Report from Eastern Europe country- Lithuania. BMC Surg. 2017; 17: 108.

4. Domingos P. A few useful things to know about machine learning Communications of the ACM. 2012; 55: 78-87.

5. Verhoef PC, Kooge E, Walk N. Creating Value with Big Data Analytics: Making Smarter Marketing Decisions. London: Routledge. 2016.

6. Power DJ. What is the "true story" about data mining, beer and diapers? DSS News.

7. Sadoughi F, Valinejadi A, Shirazi MS, Khademi R. Social Network Analysis of Iranian Researchers on Medical Parasitology: A 41 Year Co-Authorship Survey. Iran J Parasitol. 2016; 11: 204-212.

8. Osareh F, Khademi R, Rostami MK, Shirazi MS. Co-authorship Network Structure Analysis of Iranian Researchers' scientific outputs from 1991 to 2013 based on the Social Science Citation Index (SSCI). Collnet J Scientometr Info Manag. 2015; 8: 263-271.

9. Liu X, Bollen J, Nelson ML, Van de Sompel H. Co-authorship networks in the digital library research community. Info Process Manag. 2005; 41: 14621480 .

10. Luo L, Li L, Hu J, Wang X, Hou B, Zhang T, et al. A hybrid solution for extracting structured medical information from unstructured data in medical records via a double-reading/entry system. BMC Med Inform Decis Mak. 2016; 16: 114.

11. Knoblock CA, Lerman K, Minton S, Muslea I. Accurately and reliably extracting data from the web: a machine learning approach. In: Intelligent exploration of the web. New York: Springer. 2003; 275-287.

12. Ohno-Machado L. Data-driven informatics tools targeting patients and providers. J Am Med Inform Assoc. 2016; 23: 1039

13. Ohno-Machado L. Advancing healthcare and biomedical research via new data-driven approaches. J Am Med Inform Assoc. 2017; 24: 471.

14. Shah NH, Tenenbaum JD. The coming age of data-driven medicine: translational bioinformatics' next frontier. J Am Med Inform Assoc. 2012; 19: $2-4$

15. deNooy W, Mrvar A, Batagelj V. Exploratory Social Network Analysis With Pajek: Revised and Expanded, 2nd edn. New York, NY: Cambridge University Press. 2011.

16. Phan TG, Beare R, Chen J, Clissold B, Ly J, Singhal S, et al. Googling Service Boundaries for Endovascular Clot Retrieval Hub Hospitals in a Metropolitan Setting: Proof-of-Concept Study. Stroke. 2017; 48: 1353-1361.

17. Chien TW. Google Maps on the topic of author collaboration in surgery.

18. Chien TW. Google Maps on the topic of author collaboration in surgery by clusters.

19. Chien TW. Google Maps on the topic of prominent authors in surgery.

20. Chien TW. Google Maps on the topic of MESH terms in surgery.

21. Leydesdorff L, Wagner C, Park HW, Adams J. International collaboration in science: the global map and the network.

22. Glänzel W, Schlemmer B. National research profiles in a changing Europe (1983-2003) an exploratory study of sectoral characteristics in the Triple Helix. Scientometrics. 2007; 70: 267-275.

23. Erfanmanesh MA, Rohani VA, BasirianJahromi R, Gholamhosseinzadeh Z. Investigating Scientific Collaboration of Iranian Psychology and Psychiatry Researchers. J Info Process Manag. 2013; 29: 137-163.

24. Osareh F, NorouziChakoli A, Keshvari M. Co-authorship of Iranian researchers in science, social science, art and humanities citation indexes in the web of science between 2000 and 2006. J Info Sci Technol. 2010; 25: 573-595. 
25. Hu SK, Huang J, Hong WD, Du XJ, Jin R, Lin TS. The 50 Most-cited Articles in Gastroenterology and Hepatology from Mainland China. Pak J Med Sci. 2017; 33: 215-220.

26. Coelho DH, Edelmayer LW, Fenton JE. A century of citation classics in otolaryngology-head and neck surgery journals revisited. Laryngoscope. 2014; 124: 1358-1362.

27. Chang $\mathrm{HT}$, Lin MH, Hwang $\mathrm{IH}$, Chen TJ, Lin HC, Hou MC, et al. Scientific publications in gastroenterology and hepatology in Taiwan: Ananalysis of Web of Science from 1993 to 2013. J Chin Med Assoc. 2017; 80: 80-85.
28. Liu X, Bollen J, Nelson ML, Van de Sompel H. Ucinet for Windows: Software for Social Network Analysis. Info Process Manag. 2005; 41: 1462-1480.

29. Borgatti SP, Everett MG, Freeman LC. Ucinet for Windows: Software for Social Network Analysis. Harvard, MA: Analytic Technologies. 2002

30. Bastian M, Heymann S, Jacomy M. Gephi: open source software for exploring and manipulating networks. International AAAI Conference on Weblogs and Social Media. 2009.
Austin J Surg - Volume 4 Issue 5 - 2017

ISSN : 2381-9030 | www.austinpublishinggroup.com

Chov et al. () All rights are reserved
Citation: Chien TW, Chang Y, Chow JC and Chou W. Patterns of International Author Collaboration in Surgery. Austin J Surg. 2017; 4(5): 1114 